\title{
Dietary resistant starch ameliorating lipopolysaccharide-induced inflammation associated with alteration in gut microbiome and glucagon-like peptide 1 signaling
}

\section{Simeng Qin}

Sichuan Agricultural University - Chengdu Campus

\section{Weiqiang Bai}

Sichuan Agricultural University - Chengdu Campus

\section{Todd J Applegate}

University of Georgia

\section{Keying Zhang}

Sichuan Agricultural University - Chengdu Campus

\section{Gang Tian}

Sichuan Agricultural University - Chengdu Campus

\section{Xuemei Ding}

Sichuan Agricultural University - Chengdu Campus

\section{Shiping Bai}

Sichuan Agricultural University - Chengdu Campus

\section{Jianping Wang}

Sichuan Agricultural University - Chengdu Campus

Li LV

Sichuan Agricultural University - Chengdu Campus

\section{Huanwei Peng}

Sichuan Agricultural University - Chengdu Campus

\section{Yue Xuan}

Sichuan Agricultural University - Chengdu Campus

Qiufeng Zeng ( $\nabla$ zqf@sicau.edu.cn )

Institute of animal nutrition, Sichuan Agricultural University

\section{Research Article}

Keywords: Resistant starch, Intestinal integrity, Microbiota, GLP-1, Inflammatory response

Posted Date: February 10th, 2022 
DOI: https://doi.org/10.21203/rs.3.rs-1285239/v1

License: (c) (1) This work is licensed under a Creative Commons Attribution 4.0 International License. Read Full License 


\section{Abstract \\ Background}

Consumption of resistant starch (RS) has been associated with various intestinal and systemic health benefits, but knowledge of its effects on intestinal health and inflammatory response in stressed birds is limited. Here, we examined how dietary RS supplementation modulated inflammatory severity induced by lipopolysaccharide (LPS) in meat ducks.

\section{Results}

LPS administration at 14,16 , and $18 \mathrm{~d}$ (chronic challenge) decreased body weight and glucagon-like peptide 1 receptor (GLP-1R) with higher intestinal permeability and inflammation, evidenced by higher pro-inflammatory cytokine levels. Dietary RS supplementation enhanced Claudin-1 and GLP-1R expression, along with lower level of inflammatory factors in both ileum and serum. Microbiome analysis showed that RS treatment shifted microbial structure reflected by enriched the proportion of Firmicutes, Bifidobacterium, Ruminococcus, etc. Dietary RS addition also significantly increased the concentrations of propionate and butyrate during chronic LPS challenge. Further, handling LPS-challenge only at $14 \mathrm{~d}$ (acute challenge) confirmed LPS elevated the concentration of endotoxin and inflammatory cytokines in serum, accompanying with upregulated Toll Like Receptor 4 (TLR4) transcription. Analogous to GLP-1 agonist liraglutide, dietary RS administration decreased endotoxins and inflammation cytokines, whereas it numerically increased serum GLP-1 level and upregulated the GLP-1 synthesis related genes expression. Meanwhile, dietary RS supplementation suppressed the acute LPS challenge-induced TLR4 transcription.

\section{Conclusions}

These data suggest that dietary RS supplementation could attenuate the LPS-induced inflammation as well as intestinal injury of meat ducks, which might involve in the alteration in gut microbiota, SCFAs production and the signaling pathways of TLR4 and GLP-1/GLP-1R.

\section{Introduction}

The intestine of animals and humans serves as the bridge for connecting intraluminal and enteric external milieu, which mainly functioned to prevent pathogenic entities invasion and maintain intestinal homeostasis. During normal physiological activities, some endotoxins and toxins produced by colonized bacterial in intestine usually can be degraded and cleaved to non-toxic fragments by innate immune cells $[1,2]$. Nevertheless, once the organism stuck in pathological conditions, the interrupted intestinal wall allow the pathogenic bacteria to tissue, the specific molecular patterns in microbial components could been recognized by some immune factors such as toll-like receptors (TLRs) and induce the transcription 
of specific genes involved in pro-inflammatory and anti-bacterial responses [3]. These dysregulated immune responses due to intestinal disequilibrium may contribute to the occurrence of inflammatory related diseases including inflammatory bowel disease, sepsis, endotoxemia [4-6]. Modern meat ducks are no exception, various factors especially genetic selective pressure, environmental stress, bacterial infection, and immunological stress result in increasingly common incidence in the intestinal inflammation of birds [7]. The destroyed gut barrier integrity and dysbacteriosis could induce the systemic and intestinal inflammation [8]. In practically the early stage of growth and intestinal development, which is an important period relative to optimizing digestive efficiency and performance [7], the disorder of intestinal homeostasis in this phase has been noticed to associated with irreversibly weakened growth performance. Therefore, enhancing gut barrier integrity and reducing inflammation that are supposed to be an effective way for animal growth and physical health maintenance.

Currently, some functional feed ingredients targeting gut microbiota are acknowledged to counteract intestinal inflammation [9] and might be alternatives for antibiotics to product the antibiotic-free animal production in domestic birds. Resistant starch (RS), a kind of natural prebiotics exists in most common feed ingredients, is quite suitable for being supplemented in diets to maintain animal health. The most typical beneficial functions exerted by dietary RS is to enhance short chain fatty acids (SCFAs)

production through optimizing gut microbiota in hindgut because of its relatively resistant to digestion in small intestine by starch degradation enzyme produced by host $[10,11]$. Our previous studies showed that diet with $12 \%$ raw potato starch (RPS, Type $\otimes$ RS) could improve intestinal morphology and enhance intestinal barrier function through upregulating the transcription of tight junction proteins (TJPs) in both ileum and cecum, as well as it also increased the gut microbial diversity and the abundance of beneficial bacteria including Fecalibacterium and Subdoligranulum in cecum of meat ducks $[12,13]$. Accumulating evidence from rodent studies indicated that RS supplemented diet are likely to alleviate tissue damage, including but not limited to intestine, during inflammatory stress by modulating inflammatory cytokines and gut microbiota [14-17]. In addition to the metabolites such as SCFAs, glucagon-like peptide-1 (GLP1) secretion might be a key contributor to the beneficial effects on the gut [18]. GLP-1 is a gut peptide secreted in enteroendocrine $L$ cells located predominantly in the ileum and colon. It has been shown to against intestinal injury and promote gut growth via binding its receptor (GLP-1R) [19, 20]. Activation of GLP-1R is also associated with anti-inflammatory effects [21, 22]. It is reasonable to assume that dietary RS could alleviate intestinal inflammation from LPS challenge through GLP-1/GLP-1R signaling in meat ducks.

The objective of the current study, therefore, was to investigate the effects of dietary RS supplementation in the form of RPS on intestinal barrier integrity, gut microbiota, as well as systemic and intestinal inflammatory response in lipopolysaccharide (LPS)-challenged meat ducks. Of note, the SCFAs production and GLP-1/GLP-1R signaling as potential mechanism underlying the relationship between the improved intestinal status and dietary RS manipulation were also evaluated. Our hypothesis was that dietary RS supplementation would protect against LPS-induced intestinal damage and abnormal release of inflammatory cytokines of meat ducks through SCFAs production and GLP-1/GLP-1R signaling under immunological stress. 


\section{Materials And Methods}

The RS was used from RPS with a $57 \%$ RS content (Windmill @ potato starch, The Netherlands). LPS was purchased from Sigma-Aldrich (From Escherichia coli serotype 055: B5, MO, USA). GLP-1R agonist, liraglutide (Selleck, S8256). Birds were raised in temperature- and humidity-controlled room, and allowed to access water and feed freely throughout experiments. The ducklings were vaccinated at $1 \mathrm{~d}$ of age against Newcastle Disease and Infectious Bronchitis at the hatchery facilities. The basal diet was formulated to meet the requirement of meat ducks according to National Research Council (NRC, 1994). RS diet contained 12\% RPS based on our previous work [12]. Experimental diets shown in Table S1\&2.

\section{Birds and study design}

To evaluate the response of intestinal status to chronic or acute LPS challenge and dietary RS supplementation. In the chronic LPS challenge, 240 1-d-old meat ducks were randomly assigned to control, LPS challenge (LPS), and LPS challenge with fed 12\% RPS diet (LPS + RS) group. After 12-h feed deprivation, the ducks were intraperitoneally injected with either $1 \mathrm{mg} / \mathrm{kg}$ body weight (BW) of LPS or sterile saline at 14, 16, and $18 \mathrm{~d}$ (Fig. S1A). The routes of LPS injection were identical to our previous study [23]. Four hours after injecting on $18 \mathrm{~d}, 1$ duck with a weight closest to the pen average was selected for blood samples, then centrifuged at $3,000 \mathrm{~g}$ for $15 \mathrm{~min}$ at $4^{\circ} \mathrm{C}$ to gain serum or plasma, and subsequently the distal ileal, ileal mucosa and cecal digesta were collected for historical analysis, intestinal integrity evaluation, SCFA determination, and microbial analysis, respectively. For the acute LPS challenge, a total of 144 1-d-old Cherry Valley meat ducks were randomly assigned to control, LPS, LPS + RS, or LPS challenge with liraglutide ( $100 \mu \mathrm{g} / \mathrm{kg}$ BW, LPS + Liraglutide), respectively. At day 14 , birds were injected intraperitoneally with either $2 \mathrm{mg} / \mathrm{kg}$ BW of LPS or sterile saline (Fig. S1B). At $4 \mathrm{~h}$ after injecting, 1 bird from each pen was selected for blood, distal ileal, and ileal mucosa were collected, then snap frozen in liquid nitrogen and stored at $-80^{\circ} \mathrm{C}$ for further analysis.

\section{Histological analysis}

The fixed distal ileum using $10 \%$ neutral-buffered formalin for $24 \mathrm{~h}$ was embedded in paraffin. Sections of 4-mm thickness were subjected to hematoxylin and eosin staining (H\&E). Images were taken from a microscope (BA400Digital, Mike Audi Industrial Group Co., Ltd., China) and were analyzed with Image-Pro Plus 6.0 (Media Cybernetics, Silver Spring, MD, USA).

\section{Immunofluorescence analysis}

The GLP-1R protein of ileum was determined by immunofluorescence. The $4 \%$ paraformaldehyde-fixed samples were rehydrated in PBS, subjected to antigen retrieval with ethylene diamine tetraacetic acid buffer, and then blocked with $3 \%$ bovine serum albumin prior to incubation with mouse monoclonal antibodies to GLP-1R (1:100, sc-390774, Santa Cruz Biotechnology Inc., Dallas, TX, USA) overnight at $4^{\circ} \mathrm{C}$. Slides were then detected with Cy3 conjugated Goat Anti-mouse IgG (GB21301, Servicebio Technology 
Co., Ltd, Wuhan, China) for $1 \mathrm{~h}$ at room temperature in the dark, and the nuclei were stained with 4'-6diamidino-2-phenylindole (DAPI) for $10 \mathrm{~min}$. All slides were finally examined for fluorescence using a confocal scanning microscope (NIKON ECLIPSE TI-SR), and images were taken with the NIKON DS-U3 software. The proportion of GLP-1R positive cells was determined by counting cells in each of three sections from each of 6 ducks.

\section{Intestinal permeability determination}

Ducks from chronic LPS challenge trial were used to determinize the intestinal permeability using fluorescein isothiocyanate dextran (FITC-d, 4 kDa, Sigma, USA), an indicator to examine barrier function. On $\mathrm{d} 18,1$ bird each pen close to the pen average weight were selected, and all ducks received orally FITC$\mathrm{d}(4.16 \mathrm{mg} / \mathrm{kg})$. The serum was collected at $2.5 \mathrm{~h}$ post FITC-d administration and the fluorescence was measured at $485 \mathrm{~nm}$ excitation and $528 \mathrm{~nm}$ emission (BioTek Instruments, Winooski, VT). The FITCdextran concentrations were determined from standard curves generated by the serial dilution of FITC-d.

\section{Measurement of GLP-1 in plasma and cytokines in serum and ileum}

Cytokines of interleukin-1 $\beta$ (IL-1 $\beta$ ), IL-6, IL-17, and tumor necrosis factor-a (TNF- $\alpha$ ) or IL-4 concentrations in serum and ileal mucosa, as well as GLP-1 concentration in plasma were measured using enzymelinked immunosorbent assay (Meimian Biotechnology Co., Ltd, Jiangsu, China). Endotoxins concentration was analyzed by a commercially available Chromogenic End-point Tachypleus Amebocyte Lysate (CE TAL) kit (Nanjing Jiancheng Bio-Engineering Institute Co., Ltd, China) and expressed as EU/ml. All assays were performed as described by the manufacturer's instructions and done in duplicate.

\section{Gene expression assays}

Total RNA was extracted using a Trizol reagent (TaKaRa, China) from frozen ileal mucosa samples following the manufacturer's instructions. RNA integrity was tested by a $1 \%$ agarose gel electrophoresis. Real-time PCR was performed on ABI QuantStudio ${ }^{\text {TM }} 6$ Flex system (Applied Biosystems, CA, USA). The primer sequences for the target genes designed using Primer 3 (Table S3). Relative gene expression was quantified by normalizing to the expression of glyceraldehyde-3-phosphate dehydrogenase (GAPDH) and $\beta$-actin.

\section{Cecal SCFAs analysis}

Approximately $0.5 \mathrm{~g}$ of cecal digesta was diluted with $2 \mathrm{~mL}$ of ultrapure water mixed with a uniform. The solution was left for $30 \mathrm{~min}$ and then centrifuged at 3,000 $\times \mathrm{g}$ for $15 \mathrm{~min}$. Supernatants $(1 \mathrm{~mL})$ were mixed with $0.2 \mathrm{~mL}$ ice-cold $25 \%(\mathrm{w} / \mathrm{v})$ metaphosphoric acid solution, and incubated at $4{ }^{\circ} \mathrm{C}$ for $30 \mathrm{~min}$, followed by $11,000 \times \mathrm{g}$ centrifugation for $10 \mathrm{~min}$. The SCFA contents including acetate, propionate, and butyrate were separated and determined by gas chromatograph (Varian CP-3800, USA).

\section{Gut microbiome sequencing}


Total DNA was extracted from cecal content with a DNA stool mini kit (Qiagen, Valencia, CA, United States). After determining the DNA concentration and integrity, an amplicon sequencing library was constructed based on the PCR-amplified V4 variable regions of 16s rDNA, and paired-end sequenced on an Illumina HiSeq 2500 platform (BGI, Shenzhen, China). The obtained sequences were processed using FLASH (v1.2.11) and USEARCH (v7 .0.1090) for alignment and clustering. The final reads were clustered as operational taxonomic units (OTUs) with a $97 \%$ similarity threshold. The OTUs were further assigned to species level using the RDP classifer (v2.2) based on the Greengene database (V201305) of full-length 16S rRNA sequences. Partial least-squares discrimination analysis (PLS-DA) was performed by $R$ package mixOmics.

\section{Statistical analysis}

The data obtained were analyzed by the Shapiro-Wilk and Levene's test to assess normal distribution and homogeneity of variances. Analyses were done using unpaired Student's $t$-tests and the Mann-Whitney $U$ test for normally or non-normally distributed datasets, respectively in Graphpad Prism (GraphPad Software, San Diego, CA, USA), as indicated. For data of microbiota, significant genera were determined by R (v3.4.1) based on either Kruskal-Wallis test or Wilcox-test. Spearman correlation analysis was used to explore the correlation between gut bacteria and gene expression of cytokines. Differences with $P \leq$ 0.05 were considered significant.

\section{Results}

\section{Dietary RS inclusion improved BW and intestinal barrier during chronic LPS challenge}

Prior to LPS challenge, RS inclusion significantly increased $(P<0.05)$ ducks' BW at $14 \mathrm{~d}$. Subsequently, the BW at $18 \mathrm{~d}$ was notably decreased by chronic LPS-challenged, which was reversed due to dietary RS treatment (both $P<0.01$; Figure 1A). Regarding intestinal barrier, histologic examination showed that ileum form the control group presented a regularly arranged villi and intact epithelial structure (Fig. 1B). LPS injection resulted to obviously exfoliated ileal mucosa, disorderly arranged and broken villi and infiltrated with inflammatory cells, whereas the diet with RS alleviated ileal injury, showed by basically intact epithelia and slightly inflammatory cell infiltration after chronic LPS-challenged (Fig. 1B).

The outcomes from FITC-d and endotoxins, both biomarkers used to reflect intestinal permeability, suggested that the concentrations of FITC-d and endotoxins in serum were respectively significantly $(P<$ $0.01)$ or slightly $(P=0.089)$ elevated in chronic LPS-challenged group than the control group, but them were numerical decrease by RS supplementation (Fig. 1C, D). Moreover, the genes encoding intestinal barrier were determined and showed that chronic LPS challenge significantly decrease $(P<0.01) \mathrm{mRNA}$ expression levels of Occludin and Claudin-1 in ileum as compare with the control group, and the altered mRNA abundance of Claudin-1 was remarkably reversed with dietary RS administration $(P<0.01$;

Fig. 1E). 


\section{Gut microbiota and cecal SCFA concentrations response to chronic LPS challenge and dietary RS supplementation}

As illustrated in Figure 2A, there were no significant changes in individual SCFAs by challenging LPS ( $P$ > 0.05). Dietary RS supplementation significantly increased the concentrations of propionate and butyrate during chronic LPS challenge $(P<0.05)$. With regarding to the gut microbiota, the composition was apparently affected by experiment manipulation, indicated by totally separated from treatments (Fig. 2B). More specifically, except increased the proportion of Lachnospira and Mucispirillum, the chronic LPS injection failed to change the abundance of Firmicutes, Bacteroides, Bifidobacterium, and Ruminococcus compared to control group. However, diet with RS increased the proportion of Firmicutes, and thus increased the radio of Firmicutes to Bacteroides, along with higher abundance of Bifidobacterium, Mucispirillum, and Ruminococcus following the LPS-challenged (Fig. 2C-I).

\section{Dietary RS supplementation alleviated the inflammatory response induced by chronic LPS challenge might associate GLP-1 and gut microbiota}

Concerned the ileal and systemic inflammatory response, LPS challenge induced higher concentration of serum TNF-a relative to control group, which was normalized by supplementing dietary RS $(P<0.01$; Figure 3A). However, experiment treatments did not alter the content of IL-1 $\beta$ and IL-17 in serum $(P>0.05$; Fig. 3B, C). Reflecting to ileum, the mRNA levels of TNF-a, Interferon- $\gamma(I F N-\gamma), I L-1 \beta, I L-4$ and $I L-17$ were increased in chronic LPS administration $(P<0.05)$, and reversed their increased levels by dietary RS (Fig. 3D).

We further analyzed the potentially associations between inflammatory action and GLP-1 signaling and gut microbiota. The result of immunofluorescence analysis for GLP-1R showed that which was decreased by LPS challenged tended to decrease the GLP-1R expression $(P=0.08)$, and then diet supplemented of RS upregulated the abundance of GLP-1R in ileum ( $P=0.089$; Fig. 4A, B). In addition, alterations in bacteria were also associated with one or more inflammatory response factors based on Spearman's correlation coefficients (Fig. 4C). For instance, at genera levels, both IL-4 and TLR4 were negatively correlated with Megamonas. IL-4 was also significantly negatively correlated with some healthassociated gut bacteria, including Bifidobacterium, Collinsella and Ruminococcus. TNFa was negatively associated with the abundance of Odribacter. $I L-1 \beta$ and $I L-17$ are positively associated with Adlercreutzia and Sphingomonas, respectively. IFNY associated positively with Anaerostipes, Bacteroides, Eggerthella and Phascolarctobacterium, and most notably Sutterella and Bilophila, while negatively with Prevotella.

\section{Dietary RS improved ileal integrity and decreased inflammation response during acute LPS challenge}

To further explore the role of GLP1/GLP-1R signaling in the beneficial effect of dietary RS attenuating intestinal inflammation induced by LPS challenge. The model of acute LPS challenge and GLP-1R agonist liraglutide were used, and the results showed that dietary RS inclusion did not significant influence the BW in acute LPS challenged ducks among treatments (Fig. S1C). Histologic examination revealed that ileum of ducks in control groups presented a regularly arranged villi and intact epithelial 
structure, However, heavily infiltrated with inflammatory cells and partially exfoliated ileal mucosa were observed in LPS group, which were mitigated by RS supplementation or liraglutide injection, evidenced by basically intact epithelia with slightly inflammatory cell infiltration after acute LPS-challenged (Fig. 5A). The higher serum endotoxins level suggested an increase in gut permeability due to acute LPS challenge group when compared with that in control $(P<0.05)$. Both liraglutide injection and dietary RS supplementation reduced the concentration of serum endotoxins to varying degrees (Fig. 5B).

Moreover, acute LPS challenge also elevated genes expression of TNF- $a(P=0.083)$ and $T L R 4(P<0.05)$ in ileum as compared to the control, and dietary RS supplementation markedly reversed TLR4 mRNA level (Fig. 5C). Meanwhile, dietary RS and liraglutide treatment were significantly lowered IL-6 or promoted IL10 transcriptional levels (both $P<0.05$ ) relative to LPS-challenged group (Fig. 5C). In serum, acute LPS challenge increased the concentration of TNF- $a$, IL- $1 \beta$, and IL- 6 as compared with control group, and subsequently were suppressed by the administration with liraglutide injection or dietary RS (Fig. 5D-F).

\section{GLP-1/GLP-1R signaling responses to dietary RS addition during acute LPS challenge}

As presented in Figure 6A, although acute LPS change did not significantly change the concentration of plasma GLP-1, administrated with dietary RS and liraglutide result in a $32 \%$ and $24 \%$ increase in terms of serum GLP-1 content compared with LPS group, respectively (Fig. 6A). The GLP-1 synthesis related genes expression including proprotein convertase subtilisin/kexin type (Pcsk1) and solute carrier family 5 member 1 (S/c5al), as well as GLP-1R were also determined, and the data suggested that acute LPS challenge not notably affect the GLP-1R and S/c5a/ genes expression, but tended to increase the abundance of Pcsk1 mRNA (Fig. 6B-D). When compared to LPS group, liraglutide injection significantly increased GLP-1R and Pcsk1 mRNA level (Fig. 6B, C). Also, the diet supplemented with RS upregulated that transcription of Pcsk1 and S/c5al in ileum (Fig. 6C, D)

\section{Discussion}

Intestinal inflammatory disease has been becoming a great threat to poultry production in non-obvious ways due to impaired gut and growth performance of birds [24, 25]. Numerous researches that included our previous study have confirmed the release of pro-inflammatory cytokines and immunological stress triggered by LPS intraperitoneal injection could impair nutrients absorption and depress growth performance in domestic birds $[23,26,27]$. In the present study, the reduced BW by chronic LPS injection has yielded strong evidence for a harmful consequence on growth performance of meat ducks induced by LPS challenge. Furthermore, the compromised BW by chronic-LPS challenge at 18-d-old ducks were elevated by RS supplementation, indicating that RS exerted protective effects on performance of ducks suffered from immunological stress. Our previous study noticed that RS functioned as prebiotics to maintain intestinal health of ducks by improving barrier function and modulating microbial composition $[12,13]$, which could explain why the dietary RS ameliorated growth performance in LPS-challenged ducks. For this, intestinal morphology and permeability were further determined in the currently study. In lined with previous findings in ducks [27], the higher serum FITC-d concentration suggested that LPS 
challenge resulted in a drastic increase intestinal permeability. At the molecular level, chronic LPS challenge downregulated the expression of Claudin-1 and Occludin, both important composition of TJPs in intestinal barrier [28]. Evidence from our recently studies showed that dietary RS administration could enhanced gut barrier function of ducks in normal feeding via increasing mRNA expression of TJPs in ileum [12]. In addition, RS was also found to effective in protecting the TJPs expression in dextran sulfate sodium (DSS)-induced mice [15]. The present results are congruent with previous outcomes and indicated that dietary RS is beneficial to enhance intestinal barrier integrity under immunological tress.

It is well-known that compromised intestinal integrity might trigger aberrant inflammatory responses and in conjunction with accumulation of inflammatory mediators and further aggravating tissue damage [29]. Herein, we also compared the differences in alteration of systemic and intestinal inflammation response to LPS challenge and dietary RS supplementation. In this study, inflammatory lesions in ileum was consistently observed in LPS-challenged ducks, and dietary RS supplementation could effectively alleviate inflammatory injury in villus and crypt of ileum induced by LPS. RS provided also exerted a protective role against DSS-induced colonic inflammation as reflected by a decrease in colonic proinflammatory cytokine levels of IL-1 $\beta$, IL-6, TNF-a, and IFN- $\gamma$ in proximal or distal segments [15]. Analogously, our results in ileum showed that chronic LPS-challenged increased the expression of $I L-1 \beta$, $I L 4, I L-17$, and TNF- $a$, and acute LPS challenge upregulated the TNF- $a$ mRNA levels. As an antiinflammatory effect, dietary RS supplementation alleviated inflammatory response in ileum both in acute and chronic LPS challenge, evidenced by a noticeable reduction in transcriptional levels of those increased inflammatory cytokines mentioned above. Of note, the lipid A fraction contained in LPS can act as ligand for immune receptor TLR4 that elicits robust inflammatory responses in immune and somatic cells [30]. TLR4 is required to recruit specific adaptor proteins including MyD88 (myeloid differentiation primary response gene 88 etc., to initiate the downstream signaling to produce inflammatory cytokines [31] and the activation of TLR4 is preceded the release of inflammatory cytokines [32]. TLR4 is thus considered as a classical signaling pathway in triggering cytokine production, inflammation and adaptive immune response. Whereupon, the gene expression of TLR4 in ileum was examined to verify whether RS exerts an anti-inflammatory effect through this pathway. The present study with RS supplementation observed a remarkably reversion in LPS-induced increase in TLR4 mRNA level under acute LPS challenge. Taken together, our results support the ability of dietary RS to exhibited an excellent anti-inflammatory efficacy when suffered LPS challenge, which might involve in the suppression of TLR4 role.

Reflecting to systemic inflammation. Our outcomes from serum inflammatory cytokines showed that both acute and chronic LPS challenge increased concentrations of serum endotoxins. In addition to as indictor of intestinal permeability, endotoxin is also a sort of LPS that constituting much of the outer membrane of Gram-negative bacteria. When suffered bacterial infection, high concentrations of endotoxin in gut and many other tissues would be present [33]. According to results of researches in human subjects, endotoxin levels in plasma or serum are normally low, but elevated during infections or gut inflammation [30]. Therefore, systemic inflammation caused by LPS injection in this study may be evidenced by the elevated endotoxins in serum of ducks. Meanwhile, LPS induces inflammation indirectly via the pro-inflammatory cytokines such as TNF- $\alpha$, IL- 6 and IL-1 $\beta$ was related to increased endotoxin level 
[33]. We therefore examined a variety of serum inflammatory cytokines concentrations in the current study. The concentration of TNF-a significantly increased after chronic LPS-challenge and both the content of TNF- $a$ and IL- 6 in serum were also stimulated by acute LPS-challenge, which were reduced by dietary RS supplementation to varying degrees, implying that dietary RS supplementation contributes to normalized the release of pro-inflammatory cytokines during inflammatory infection induced by LPS challenge.

Gut microbiota is considered to be a crucial part of intestinal homeostasis and paly key role in inflammatory response. There are accumulating evidence have confirmed that RS is beneficial for modulating microbiota in hindgut [10]. As we described in our previous study, diet with $12 \%$ RPS had a higher relative abundance of Firmicutes [13]. In the present study, we also confirmed that RS supplementation resulted in a shift in the microbial structure, and there was a consistent increase in the abundance of Firmicutes and the ratio of Firmicutes and Bacteroidetes following RS diet with LPSchallenged compared to basal diet with LPS-challenged, suggesting that members of the Firmicutes had more selective advantage than members of the Bacteroides when supplementing diet with RS, as similar results were also observed in study on human with high levels of RS diet [11]. R.brommi that belongs to Ruminococcus has been shown to degrade RS, and numerous studies in human and mice have reported RS supplementation could increase the amounts of Ruminococcus and Lachnospira [11, 17, 34, 35]. Besides, following these changes, the relative abundance of Ruminococcus and Lachnospira were enriched in RS supplemented diet. These two genera are known as butyrate-producing bacteria, which was in line with the increase of butyrate in the cecal contents of ducks consuming RS diet. In addition, SCFAs is known as the microbiota-derived metabolites produced in cecum and colon, which are the signatures of the gut microbiota and contribute to modulate intestinal immune activity and inflammatory responses [36]. Our results of SCFAs showed that dietary RS supplementation elevated the concentration of propionate and butyrate, which was in agreement with our previous study [13], in which diet supplemented with $12 \%$ RPS could increase SCFAs contents in normal feeding experiment. To link the gut microbiota with the inflammatory markers, a correlation analysis was integrated and suggested that there were various bacteria significantly associated with these inflammatory cytokines and mediators. For example, the RS-degrading organisms Bifidobacterium and Ruminococcus were negatively associations with IL-4. Bilophila was positively related to IFNY. Pathobiont Bilophila was associated with aggravates metabolic dysfunctions in mice [37]. These results indicated that alterations in cecal microbial composition derived by dietary RS supplementation may play a pivotal role in alleviating inflammatory responses induced by LPS challenge.

Emerging evidence demonstrated that inflammatory stimuli increased the GLP-1 secretion, which was found increased in rodents with experimental inflammation induced by LPS challenge [21, 38, 39]. In turn, GLP-1 could modulate inflammation in multiple sites such as blood vessels [40]. Considering the inseparable relations between dietary RS treatment and GLP-1 secretion [18]. The GLP-1R agonist liraglutide was used to dig the role of RS in inflammatory response through GLP-1 production. GLP-1 exerts anti-inflammatory capacities through directly reduce inflammation in organs expressing the GLP$1 \mathrm{R}$, and/or by GLP-1 targets GLP-1R expressed on circulating immune cells indirectly reduce 
inflammation [40]. In the present study, dietary RS addition alleviated pro-inflammatory cytokine levels including TNF- $\alpha$, IL- 6 and IL-1 $\beta$, which were similar to the anti-inflammatory effect exerted by liraglutide in serum. However, the downregulated mRNA abundance of IL-6 and TLR4 and comparable antiinflammatory cytokine $I L-10$ transcription by dietary RS administration was incompatible with the role of liraglutide. To further illustrate this, serum GLP-1 content and the GLP-1 synthesis related genes including Pcsk1 and S/c5a/ were determined in the present study. The data showed that GLP-1 secretion was consistently numerically increased in dietary RS supplementation and liraglutide administration during acute LPS challenge, and the diet supplemented with RS upregulated the transcription of S/c5al, which was analogous to it in liraglutide injection. Therefore, this improvement in intestinal and systemic inflammation from our data may indirectly reflect long-term RS supplementation plays as a stimulator in activation of GLP-1/GLP-1R signaling. Indeed, previous research showed that augmentation of various cytokines gene expression in the jejunum by continuously GLP-1R agonist Ex-4 treated was rapidly returning to normal by $24 \mathrm{~h}$, which also indicated that GLP-1 could suppresses a pro-inflammatory cytokine program, and the action requiring a functional GLP-1R (Yusta et al. 2015). Collectively, our findings provide a new angle of view for regulating host and intestinal inflammation by dietary RS treatment through GLP-1/GLP-1R signaling.

This study raises three new question need to be elaborated. The first is the response of gut microbiota to chronic LPS challenge. It was established that inflammation is triggered by LPS derived from the gut microbiota. However, in this study, except increased the proportion of Lachnospira and Mucispirillum, the chronic LPS injection failed to change the abundance of Firmicutes, Bacteroides, Bifidobacterium, and Ruminococcus. The second is the difference of GLP-1R expression between acute and chronic LPS challenge. Herein, chronic LPS challenge decreased the expression of GLP-1R using histometric method, whereas no apparently change was observed in acute LPS injection via RT-PCR determination. The third is LPS challenge model. The assessment for the link of dietary RS, inflammation, and GLP1/GLP-1R signaling was conducted using acute but not chronic LPS challenge, it is possible that the responses of GLP1/GLP-1R signaling to acute and chronic LPS challenge are complicated and changeable. Therefore, some of inaccurate conclusion highlighted the roles of dietary RS addition in modulating LPS-induced inflammatory response might be included in this study. Further researches would be essential to exclude this possibility.

\section{Conclusion}

In conclusion, the research has revealed a substantial efficacy of dietary RS supplementation in ameliorating inflammatory severity of LPS-induced in meat ducks, which were associated with improved intestinal integrity and gut microbiota, as well as SCFAs production. Moreover, this anti-inflammatory effect exerted by dietary RS in LPS challenge possibly also involves the TLR4 and GLP-1/GLP-1R signaling pathway. These results will bring a valuable strategy of nutritional immunity for protecting host by exploiting dietary RS supplementation ways to against immunological stress for domestic birds and human. 


\section{Abbreviations}

DSS

dextran sulfate sodium

GLP-1

glucagon-like peptide-1

GLP-1R

glucagon-like peptide 1 receptor

TLR4

IFN- $\gamma:$ Interferon- $\gamma$

IL-1 $\beta$

interleukin-1 $\beta$

LPS

lipopolysaccharide

Pcsk1

proprotein convertase subtilisin/kexin type

Toll Like Receptor 4

RPS

raw potato starch

RS

Resistant starch

SCFAs

short chain fatty acids

Slc5al

solute carrier family 5 member 1

TJPs

tight junction proteins

TLRs

toll-like receptors

TNF-a

tumor necrosis factor alpha.

\section{Declarations}

\section{Authors' contributions}

Simeng Qin \& Weiqiang Bai: Animal trial, Data collection and evaluation, Laboratory and Statistical analysis, Writing, Todd J Applegate: Critical manuscript review, Keying Zhang: Methodology, Data collection and evaluation, Laboratory analysis, Writing, Gang Tian: Data collection and Manuscript review, Xuemei Ding: Data evaluation, Manuscript review, Shiping Bai: Data collection and evaluation, Statistical analysis, and Writing, Jianping Wang: Data evaluation, Manuscript review, Writing, Li Lv: Laboratory 
analysis, Data collection, Huanwei Peng: Manuscript review, Yue Xuan: Animal trial, Laboratory analysis, Data collection, Quifeng Zeng: Study design, Feed formulation, Data evaluation, Critical manuscript review.

\section{Funding}

This work was supported by the National Natural Science Foundation of China (31772622), China Agriculture Research System (CARS-42-10), National Key R \& D Program of China (2017YFD0502004), "111" project of Foreign Experts Affairs of China, Sichuan Agricultural University 211 Foundation.

\section{Ethics approval}

All procedures involving live animals were verified and approved by the Institutional Animal Care and Use Committee of Sichuan Agricultural University (Animal Use Protocol \#SAUPN-19-05).

\section{Consent for publication}

Not applicable.

\section{Availability of data and material}

All data generated or analysed during this study are included in this manuscript and its supplementary information files.

\section{Competing interests}

We declare that we have no financial and personal relationships with other people or organizations that can inappropriately influence our work, there is no professional or other personal interest of any nature or kind in any product, service and/or company that could be construed as influencing the content of this paper.

\section{Acknowledgements}

Not applicable.

\section{References}

1. Nguyen-Lefebvre AT, Horuzsko A. Kupffer Cell Metabolism and Function. J Enzymol Metab. 2015; 1 (1):101. PMCID: PMCPMC4771376.

2. Bertók L. Endotoxins and endocrine system. Domest Anim Endocrinol. 1998;15(5):305-8.

3. Akira S, Takeda K. Toll-like receptor signalling. Nat Reviews Immunol. 2004;4(7):499-511.

4. Asari Y, Majima M, Sugimoto K, Katori M, Ohwada T. Release site of TNF alpha after intravenous and intraperitoneal injection of LPS from Escherichia coli in rats. Shock. 1996;5(3):208-12. 
5. Lelubre C, Vincent JL. Mechanisms and treatment of organ failure in sepsis. Nat Rev Nephrol. 2018;14(7):417-27.

6. Bain CC, Mowat AM. Macrophages in intestinal homeostasis and inflammation. Immunol Rev. 2014;260(1):102-17.

7. Lilburn MS, Loeffler S. Early intestinal growth and development in poultry. Poult Sci. 2015;94(7):1569-76.

8. Hooper LV, Littman DR, Macpherson AJ. Interactions between the microbiota and the immune system. Science. 2012;336(6086):1268-73.

9. Vitetta L, Vitetta G, Hall S. Immunological Tolerance and Function: Associations Between Intestinal Bacteria, Probiotics, Prebiotics, and Phages. Front Immunol. 2018;9:2240.

10. DeMartino P, Cockburn DW. Resistant starch: impact on the gut microbiome and health. Curr Opin Biotechnol. 2020;61:66-71.

11. Maier TV, Lucio M, Lee LH, VerBerkmoes NC, Brislawn CJ, Bernhardt J, et al. Impact of Dietary Resistant Starch on the Human Gut Microbiome, Metaproteome, and Metabolome. mBio. 2017;8(5):e01343-17.

12. Qin SM, Zhang KY, Ding XM, Bai SP, Wang JP, Zeng QF. Effect of dietary graded resistant potato starch levels on growth performance, plasma cytokines concentration, and intestinal health in meat ducks. Poult Sci. 2019;98(9):3523-32.

13. Qin S, Zhang K, Applegate TJ, Ding X, Bai S, Luo Y, et al. Dietary administration of resistant starch improved caecal barrier function by enhancing intestinal morphology and modulating microbiota composition in meat duck. Br J Nutr. 2020;123(2):172-81.

14. Bai Y, Li Y, Marion T, Tong Y, Zaiss MM, Tang Z, et al. Resistant starch intake alleviates collageninduced arthritis in mice by modulating gut microbiota and promoting concomitant propionate production. J Autoimmun. 2021;116:102564.

15. Shinde T, Perera AP, Vemuri R, Gondalia SV, Beale DJ, Karpe AV, et al. Synbiotic supplementation with prebiotic green banana resistant starch and probiotic Bacillus coagulans spores ameliorates gut inflammation in mouse model of inflammatory bowel diseases. Eur J Nutr. 2020;59(8):3669-89.

16. Majumder K, Fukuda T, Zhang H, Sakurai T, Taniguchi $Y$, Watanabe $H$, et al. Intervention of Isomaltodextrin Mitigates Intestinal Inflammation in a Dextran Sodium Sulfate-Induced Mouse Model of Colitis via Inhibition of Toll-like Receptor-4. J Agric Food Chem. 2017;65(4):810-7.

17. Zhang Y, Chen L, Hu M, Kim JJ, Lin R, Xu J, et al. Dietary type 2 resistant starch improves systemic inflammation and intestinal permeability by modulating microbiota and metabolites in aged mice on high-fat diet. Aging. 2020;12(10):9173-87.

18. Keenan MJ, Zhou J, Hegsted M, Pelkman C, Durham HA, Coulon DB, et al. Role of resistant starch in improving gut health, adiposity, and insulin resistance. Adv Nutr. 2015;6(2):198-205.

19. Kissow H, Hartmann B, Holst JJ, Poulsen SS. Glucagon-like peptide-1 as a treatment for chemotherapy-induced mucositis. Gut. 2013;62(12):1724-33. 
20. Koehler JA, Baggio LL, Yusta B, Longuet C, Rowland KJ, Cao X, et al. GLP-1R agonists promote normal and neoplastic intestinal growth through mechanisms requiring Fgf7. Cell Metab. 2015;21(3):379-91.

21. Iwai T, Ito S, Tanimitsu K, Udagawa S, Oka J. Glucagon-like peptide-1 inhibits LPS-induced IL-1beta production in cultured rat astrocytes. Neurosci Res. 2006;55(4):352-60.

22. Yusta B, Baggio LL, Koehler J, Holland D, Cao X, Pinnell LJ, et al. GLP-1R Agonists Modulate Enteric Immune Responses Through the Intestinal Intraepithelial Lymphocyte GLP-1R. Diabetes. 2015;64(7):2537-49.

23. Bai WQ, Zhang KY, Ding XM, Bai SP, Wang JP, Peng HW, et al. High dietary energy content increases inflammatory markers after lipopolysaccharide challenge in meat ducks. Poult Sci. 2019;98(1):16471.

24. Tao Z, Zhu C, Xu W, Shi Z, Zhang S, Song W, et al. Riemerella anatipestifer infection affects intestinal barrier structure and immune reactions in the duck caecum. Avian Pathol. 2020;49(6):572-80.

25. Dierick E, Hirvonen OP, Haesebrouck F, Ducatelle R, Van Immerseel F, Goossens E. Rapid growth predisposes broilers to necrotic enteritis. Avian Pathol. 2019;48(5):416-22.

26. Chen Y, Zhang H, Cheng Y, Li Y, Wen C, Zhou Y. Dietary I-threonine supplementation attenuates lipopolysaccharide-induced inflammatory responses and intestinal barrier damage of broiler chickens at an early age. Br J Nutr. 2018;119(11):1254-62.

27. Liu Y, Jiang G, Huang X, Li C, Huang X, Zhang X, et al. Evaluation of serum antioxidative status, immune status and intestinal condition of Linwu duck challenged by lipopolysaccharide with various dosages and replications. Poult Sci. 2021;100(7):101199.

28. Groschwitz KR, Hogan SP. Intestinal barrier function: molecular regulation and disease pathogenesis. J Allergy Clin Immunol. 2009;124(1):3-20.

29. Xavier RJ, Podolsky DK. Unravelling the pathogenesis of inflammatory bowel disease. Nature. 2007;448(7152):427-34.

30. Gnauck A, Lentle RG, Kruger MC. Chasing a ghost?--Issues with the determination of circulating levels of endotoxin in human blood. Crit Rev Clin Lab Sci. 2016;53(3):197-215.

31. O'Neill LAJ, Bowie AG. The family of five: TIR-domain-containing adaptors in Toll-like receptor signalling. Nat Rev Immunol. 2007;7(5):353-64.

32. Mogensen TH. Pathogen recognition and inflammatory signaling in innate immune defenses. Clin Microbiol Rev. 2009;22(2):240-73.

33. Brown GC. The endotoxin hypothesis of neurodegeneration. J Neuroinflammation. 2019;16(1):180.

34. Baxter NT, Schmidt AW, Venkataraman A, Kim KS, Waldron C, Schmidt TM. Dynamics of Human Gut Microbiota and Short-Chain Fatty Acids in Response to Dietary Interventions with Three Fermentable Fibers. mBio. 2019;10(1):e02566-18.

35. Ze X, Duncan SH, Louis P, Flint HJ. Ruminococcus bromii is a keystone species for the degradation of resistant starch in the human colon. ISME J. 2012;6(8):1535-43. 
36. Maslowski KM, Vieira AT, Ng A, Kranich J, Sierro F, Yu D, et al. Regulation of inflammatory responses by gut microbiota and chemoattractant receptor GPR43. Nature. 2009;461(7268):1282-6.

37. Natividad JM, Lamas B, Pham HP, Michel ML, Rainteau D, Bridonneau C, et al. Bilophila wadsworthia aggravates high fat diet induced metabolic dysfunctions in mice. Nat Commun. 2018;9(1):2802.

38. Nguyen AT, Mandard S, Dray C, Deckert V, Valet P, Besnard P, et al. Lipopolysaccharides-Mediated Increase in Glucose-Stimulated Insulin Secretion: Involvement of the GLP-1 Pathway. Diabetes. 2014;63(2):471-82.

39. Kahles F, Meyer C, Mollmann J, Diebold S, Findeisen HM, Lebherz C, et al. GLP-1 secretion is increased by inflammatory stimuli in an IL-6-dependent manner, leading to hyperinsulinemia and blood glucose lowering. Diabetes. 2014;63(10):3221-9.

40. Drucker DJ. The Cardiovascular Biology of Glucagon-like Peptide-1. Cell Metab. 2016;24(1):15-30.

\section{Figures}

\section{Figure 1}

Dietary resistant starch (RS) reversed the chronic lipopolysaccharide (LPS)-induced the compromised body weight and the impaired intestinal barrier integrity. (A) Body weight, (B) Representative hematoxylin/eosin (H\&E) staining of ileum, Gut permeability was evaluated by (C) Fluorescein isothiocyanate (FITC)-dextran and (D) Serum endotoxins, (E) The genes encoding tight junction proteins including zonula occludens-1 (ZO-1), Occludin, and Claudin-1 in ileal mucosa were determined. Mean values with the letter $*$ are significantly different $\left({ }^{\star} P<0.05,{ }^{\star} * P<0.01\right)$.

\section{Figure 2}

Short-chain fatty acids (SCFAs) and gut microbiome in response to dietary resistant starch (RS) supplementation under chronic lipopolysaccharide (LPS) challenge. (A) Concentrations of SCFAs in cecal contents, (B) Partial least-squares discrimination analysis (PLS-DA) analysis, (C) Composition profiles of gut microbiome at phyla level among treatments revealed by 16S rRNA sequencing, Alterations of relative abundance of representative bacteria (D) Bacteroidetes and Firmicutes, and (E) calculated the ratio of Firmicutes/Bacteroidetes, (F) Lachnospira, (G) Bifidobacterium, (H) Mucispirillum, (I) Ruminococcus. Mean values with the letter * are significantly different $\left({ }^{\star} P<0.05\right)$.

\section{Figure 3}


Dietary resistant starch (RS) ameliorated the chronic lipopolysaccharide (LPS)-induced systemic and intestinal inflammation. Inflammatory cytokines (A) TNF-a, (B) IL-1 $\beta$ and (C) IL-17 concentrations in serum. Data represent means with standard deviation ( $\left.{ }^{\star *} P<0.01\right)$. (D) A heat map showing the mRNA expression of inflammatory cytokines in ileum. IL, interleukin, TNF, tumor necrosis factor, IFN, interferon, TLR, toll like receptor. Mean values with the letter * (compared with the control group)/\# (compared with the LPS group) are significantly different $\left({ }^{\star}, \# P<0.05,{ }^{\star *}, \# \# P<0.01\right)$.

\section{Figure 4}

(A) Expression and (B) distribution of glucagon-like peptide-1 receptor (GLP-1R) expression response to dietary resistant starch (RS) administration under chronic lipopolysaccharide (LPS) challenge were assessed using immunofluorescence (IF) staining. (C) Spearman correlation analysis of the cecal microbiota and ileal inflammatory cytokines gene expression. $P$-values are depicted from blue to red, where red represents a positive correlation and blue represents a negative correlation.

\section{Figure 5}

Dietary resistant starch (RS) and liraglutide administration ameliorated the acute lipopolysaccharide (LPS)-induced systemic and intestinal inflammation and the compromised intestinal permeability. (A) Representative hematoxylin/eosin (H\&E) staining of ileum, (B) Gut permeability was evaluated by serum endotoxins, (C) A heat map showing the mRNA expression of inflammatory cytokines in ileum. IL, interleukin, TNF, tumor necrosis factor, IFN, interferon, TLR, toll like receptor. Mean values with the letter * (compared with the control group)/\# (compared with the LPS group) are significantly different $\left({ }^{*}, \# P<\right.$ 0.05 , **,\#\# $P<0.01$ ). Inflammatory cytokines (D) TNF-a, (E) IL-1 $\beta$ and (F) IL-6 concentrations in serum. Mean values with the letter * are significantly different $\left({ }^{\star} P<0.05\right)$.

\section{Figure 6}

Glucagon-like peptide (GLP) -1 signaling response to dietary resistant starch (RS) and liraglutide administration under acute lipopolysaccharide (LPS) challenge. (A) Plasma GLP-1 contents, Genes expression of (B) GLP-1R, (C) proprotein convertase subtilisin/kexin type (Pcsk1) and (D) solute carrier family 5 member 1 (S/c5a). Data represent means with standard deviation $(* P<0.05)$.

\section{Supplementary Files}

This is a list of supplementary files associated with this preprint. Click to download. 
- Supplementalmaterial.docx

Page 19/19 